\title{
Síntesis y propiedades de electrodos de níquel/grafeno para generación de hidrógeno
}

\section{Synthesis and properties of nickel/graphene electrodes for hydrogen generation}

Melisa Julieta Gomez ${ }^{1}$, Esteban Andres Franceschini ${ }^{1,2}$, Horacio Roberto Corti ${ }^{2,3}$, Gabriela Inés Lacconi ${ }^{1}$

\author{
${ }^{1}$ INFIQC-CONICET, Dto. de Fisicoquímica - Facultad de Ciencias Químicas, Universidad Nacional de Córdoba, Ciudad \\ Universitaria, 5000, Córdoba, Argentina. \\ e-mail: estebanfranceschini@yahoo.com.ar \\ ${ }^{2}$ Departamento de Física de la Materia Condensada, Centro Atómico Constituyentes, Comisión Nacional de Energía \\ Atómica, Av. Gral. Paz 1499 (B1650KNA) San Martín, Buenos Aires, Argentina. \\ ${ }^{3}$ Departamento de Química Inorgánica, Analítica y Química Física and INQUIMAE-CONICET, Facultad de Ciencias \\ Exactas y Naturales, Universidad de Buenos Aires, Ciudad Universitaria, Pabellón II, C1428EHA, Buenos Aires, Argen- \\ tina. \\ e-mail: melisa.julieta.gomez@gmail.com; glacconi@fcq.unc.edu.ar,hrcorti@cnea.gov.ar
}

\section{RESUMEN}

El empleo de nanomateriales, por ejemplo el óxido de grafeno (GO), como bloques para la síntesis de materiales híbridos, ha sido ampliamente estudiado durante los últimos años. En este trabajo se presenta un método simple y reproducible para la síntesis de electrodos híbridos de níquel/grafeno. Este método consiste en la modificación de un baño de electrodeposición convencional mediante la incorporación de escamas de GO, por lo que la síntesis puede ser industrialmente escalable.

El catalizador sintetizado fue caracterizado estructural y electroquímicamente. Los estudios de XRD no muestran cambios apreciables en el parámetro de red, aunque se observa una disminución en la relación de planos (200)/(111) relacionado con un aumento en la estabilidad del catalizador. Los espectros Raman muestran las bandas características de GO reducido en la matriz del catalizador. Asimismo, la formación de hidruros de níquel en el catalizador níquel/grafeno no es observada, a diferencia del electrodo de níquel puro.

La cinética de la reacción de desprendimiento de hidrógeno (HER) se estudió en ambos catalizadores, níquel convencional y níquel/grafeno, encontrando cambios en la actividad. Debido a que la presencia de GO en la matriz de Ni inhibe la hidruración del níquel, el catalizador híbrido presenta una mayor actividad catalítica hacia la HER.

Palabras clave: Grafeno, Níquel, Generación de hidrógeno, Espectroscopía Raman.

\section{ABSTRACT}

The use of nanomaterials, such graphene oxide (GO), as blocks for the synthesis of hybrid materials has been extensively studied in recent years. In this paper a simple and reproducible method for the synthesis of hybrid nickel/graphene electrodes is presented. This method involves the modification of a conventional electroplating nickel bath by incorporating graphene oxide flakes, so the synthesis can be industrially scalable.

The synthesized catalyst was structurally and electrochemically characterized. The XRD studies show no appreciable change in the lattice parameter, but a decrease is observed in the relationship between (200)/(111) planes related to an increase in catalyst stability. Raman spectra show the characteristic reduced GO bands on the catalyst matrix. Also, the formation of nickel hydrides in the nickel/graphene catalyst is not observed, unlike the pure nickel electrode.

The kinetics of hydrogen evolution reaction (HER) was studied in both, conventional nickel and nickel/graphene catalysts, finding changes in activity. The hybrid catalyst has a higher catalytic activity towards 
the HER and the presence of GO in the Ni matrix inhibits the nickel hydride formation with the consequent loss of catalytic activity.

Keywords: Graphene, Níckel, Hydrogen Generation, Raman Spectroscopy.

\section{INTRODUCCIÓN}

El hidrógeno ha atraído recientemente la atención como vector de energía limpia, que puede ser utilizado en pilas de combustible, o en sustitución de gas natural [14]. La producción de hidrógeno por electrólisis de agua se considera la forma de producción más limpia para la economía de hidrógeno en un futuro próximo $[3,4]$.

Uno de los enfoques utilizados para mejorar la actividad electrocatalítica de los catalizadores para reacciones electroquímicas consiste en el cambio de las propiedades extrínsecas de electrocatalizadores, por ejemplo, la modificación de la morfología de la superficie [3-6] el tamaño de partícula [3] de los electrodepósitos.

El grafeno exhibe diversas propiedades únicas, tales como la rápida movilidad de portadores de carga a temperatura ambiente $\left(2 \times 10^{5} \mathrm{~cm}^{2} \mathrm{~V}^{-1} \mathrm{~s}^{-1}\right)$, una conductividad excepcional $\left(106 \mathrm{Scm}^{-1}\right)$, gran superficie específica teórica $\left(2630 \mathrm{~m}^{2} \mathrm{~g}^{-1}\right)$, y una excelente transmitancia óptica $(\sim 97,7 \%)$ [3]. Estas propiedades destacadas son indicativas de que el grafeno tiene un gran potencial para ser un componente en la construcción de materiales de electrodos híbridos para la producción de hidrógeno.

En este trabajo se presenta un método simple y reproducible para la síntesis de un electrodo híbrido de níquel/grafeno (NiGO) para la reacción de generación de hidrógeno. En la Figura 1 se muestra una representación esquemática de la reacción de desprendimiento de $\mathrm{H}_{2}$ sobre un catalizador de NiGO. El método desarrollado consiste en la modificación directa de un baño de electrodeposición de níquel convencional (baño de Watts), sin necesidad de altas temperaturas, tiempos de reacción largos o complejos pasos de síntesis. El material híbrido sintetizado se caracterizó como electrodo para la reacción de generación de hidrógeno en medio alcalino. Todos los catalizadores sintetizados se caracterizaron antes y después de un proceso de envejecimiento corto con el fin de estudiar el decaimiento en la actividad electrocatalítica debida a la formación de hidruros de níquel superficiales.

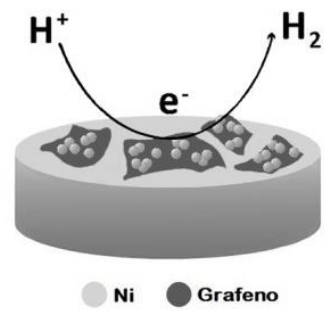

Figura 1: Representación esquemática de la reacción de desprendimiento de $\mathrm{H}_{2}$ sobre un catalizador de NiGO.

\section{MATERIALES Y MÉTODOS}

Ácido clorhídrico 36.5 - $37 \%$ (Cicarelli, PA grade), hidróxido de potasio (Anedra RA reagent), etanol $96 \%$ (Cicarelli, PA grade), sulfato de níquel (Anedra, PA grade), cloruro de niquel (Merck, PA grade), ácido borico (Merck, PA grade), y dispersión de óxido de grafeno (Graphenea Inc.) fueron utilizados en las mismas condiciones en las que fueron recibidos y sin purificaciones posteriores. Todas las soluciones y dispersiones acuosas fueron preparadas con agua Milli Q y desgasadas usando $\mathrm{N}_{2}$ de alta pureza (Indura S.A.).

\subsection{Caracterización Morfológica}

Las micrografías SEM fueron obtenidas con un FESEM Supra 40 (Zeiss Company) operando a $3 \mathrm{kV}$. Los espectros y mapeos EDX fueron obtenidos a $10 \mathrm{kV}$. Las muestras fueron obtenidas mediante el raspado de los electrodos de níquel donde fueron electrodepositados.

Los espectros Raman de los electrodos fueron adquiridos con un microscopio Raman LABRAM-HR, Horiba Jobin-Yvon con un objetivo de $100 \times$ (NA = 0.9).

Fue utilizado un láser de Ar de $488.0 \mathrm{~nm}$ con una potencia de $8.5 \mathrm{~mW}$ con el objetivo de evitar el calentamiento de la muestra originado por el láser. El área Raman iluminada en todos los experimentos fue de 
$1.0 \mu \mathrm{m}^{2}$ con una resolución espectral de $1.5 \mathrm{~cm}^{-1}$ y los espectros fueron tomados en al menos cinco zonas diferentes de cada muestra analizada, para asegurar la homogeneidad de los catalizadores.

Los difractogramas de rayos $\mathrm{X}$ fueron medidos empleando una fuente de radiación $\mathrm{Cu} \mathrm{K} \alpha(\lambda=1.5406$ $\AA$ A) usando un difractómetro PANalytical X'Pert PRO (40kV, $40 \mathrm{~mA})$, con la configuración $\theta-2 \theta$ BraggBrentano a temperatura ambiente. Un intervalo de $2 \theta$ de entre $10^{\circ}$ y $70^{\circ}$ fue utilizado, con incrementos de $0.02^{\circ}$ tomando $14 \mathrm{~s}$ por paso. El programa FULLPROF [3] fue aplicado con el objetivo de refinar la estructura cristalina obtenida por el método de Rietveld. Una forma de pseudo-Voigt fue utilizada para ajustar los datos experimentales. Los datos refinados fueron las posiciones atómicas, los parámetros de red, la forma de los picos, los parámetros térmicos isotrópicos y los factores de ocupación.

\subsection{Síntesis del Catalizador}

Los catalizadores se sintetizaron usando un baño de electrodeposición convencional de Ni preparado por disolución de $25 \mathrm{~g}$ de ácido bórico en agua a $50{ }^{\circ} \mathrm{C}, 240 \mathrm{~g}$ de sulfato de níquel hexahidratado y $25 \mathrm{~g}$ de cloruro de níquel hexahidratado para obtener $1 \mathrm{~L}$ de solución. Este baño de electrodeposición (Watts) fue utilizado para sintetizar un catalizador de níquel electrodepositado convencional, a modo comparativo.

Parte de ese baño de Ni Watts se separó para preparar un baño de Ni/grafeno para electrodepositar un catalizador híbrido Ni/GO. Para eso, una dispersión de GO de $0,08 \mathrm{~g} / \mathrm{L}$ fue sonicada durante 30 minutos con el fin de dispersar las escamas de GO. A continuación, se añadió una alícuota de $10 \mathrm{ml}$ de la dispersión de $\mathrm{GO}$ a $40 \mathrm{ml}$ del baño Watts.

La síntesis de ambos catalizadores se llevó a cabo por electrodeposición sobre un electrodo de Ni comercial (R.C. S.A. 99,9 de pureza), con una superficie circular de $0,196 \mathrm{~cm}^{2}$, que está montado en un electrodo de disco rotante (RDE, Pine Investigation Inst.; Raleigh). Los electrodos de níquel se pulieron mecánicamente con una suspensión de alúmina de $0,05 \mu \mathrm{m}$ y fueron limpiados con etanol e inmersos en $\mathrm{KOH}(1 \mathrm{M})$ y $\mathrm{HCl}(10 \% \mathrm{p} / \mathrm{p})$ durante 1 minuto, con el fin de desengrasar la superficie [3]. Ambas síntesis de catalizadores se llevaron a cabo en una celda electroquímica de dos electrodos termostatizada a $50{ }^{\circ} \mathrm{C}$, usando un electrodo de níquel masivo de alta área como contraelectrodo. Durante el proceso, para asegurar la homogeneidad del electrodepósito, el electrodo de trabajo se mantuvo con una velocidad de rotación de $1600 \mathrm{rpm}$. Por otra parte, la rotación del electrodo de trabajo facilita la difusión de los iones de Ni y las partículas de GO a la superficie del electrodo.

La corriente aplicada durante la síntesis de los dos catalizadores de Ni-Watts y NiGO fue de 0,05 $\mathrm{Acm}^{-2}$ catódica, durante $3600 \mathrm{~s}$, con el fin de obtener un espesor de depósito de alrededor de $60 \mu \mathrm{m}$. Durante la electrodeposición de ambos catalizadores el potencial transitorio alcanza alrededor de - $5 \mathrm{~V}$, lo que indica que las escamas de GO co-depositadas deben estar en un estado reducido, condiciones en las cuales el grafeno es altamente conductor [3].

Después de la síntesis, ambos catalizadores se lavaron repetidamente con agua Milli Q con el fin de eliminar los restos del baño de electrodeposición sobre la superficie del electrodo.

\section{RESULTADOS Y DISCUSIÓN}

Los catalizadores obtenidos con los diferentes baños de electrodeposición tienen diferentes propiedades ópticas. Mientras que el electrodo de Ni-Watts es gris opaco, el electrodo NiGO es negro, lo que sugiere la presencia de GO en la matriz del catalizador.

Micrografías electrónicas de barrido (SEM) se obtuvieron con el fin de analizar la morfología y la presencia de grietas en ambos catalizadores electrodepositados. Las imágenes SEM del catalizador de NiWatts muestra una estructura granular homogénea. En las imágenes SEM del electrodo de NiGO (Figura 2) se observa que la muestra tiene granos de menor tamaño, sin marcas de pulido y fracturas superficiales. Se puede ver, en particular a bajos aumentos, que las escamas de grafeno se distribuyen sobre la superficie del electrodo NiGO.

El análisis de la composición por EDX no muestra contaminación metálica de las muestras y sólo se observan trazas de $\mathrm{Al}$ y $\mathrm{Si}$, debidas al pulido mecánico. El mapeo EDX muestra una composición homogénea de Ni y C en toda la superficie de la muestra. Sin embargo, este análisis no es suficiente para la cuantificación ya que la contaminación de carbono ambiental está siempre presente, por ejemplo, de la cinta de carbono conductora utilizada para fijar las muestras al soporte. 


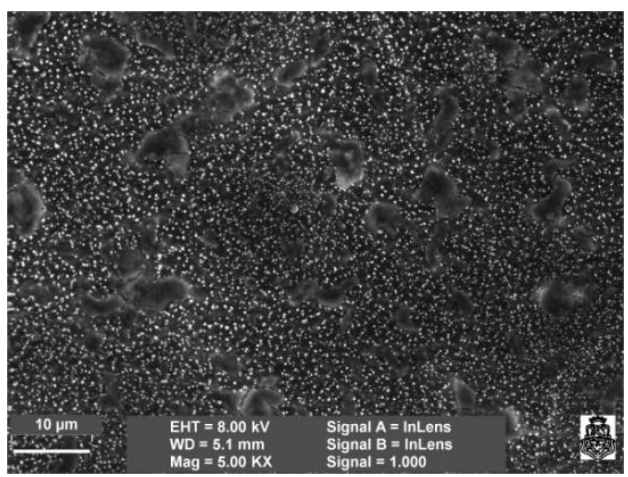

Figura 2: Micrografía SEM de una muestra de NiGO medida a $3 \mathrm{kV}$ y una magnificación de $5 \mathrm{kX}$.

Los espectros Raman registrados en ambos catalizadores se muestran en la Fig. 3, junto con el espectro de GO comercial soportado sobre una placa de silicio con fines comparativos. Los espectros obtenidos en diferentes áreas de cada muestra no muestran diferencias significativas. Los espectros del electrodo de NiWatts no muestran picos, indicando que el níquel se encuentra en estado metálico y no hay señal de hidruros de níquel, ya que estos materiales muestran bandas Raman en 3581 y $3660 \mathrm{~cm}^{-1}$ [3]. El espectro Raman del electrodo NiGO muestra las bandas características de GO, lo que evidencia la presencia de grafeno intercalado en la estructura del electrodepósito de níquel.

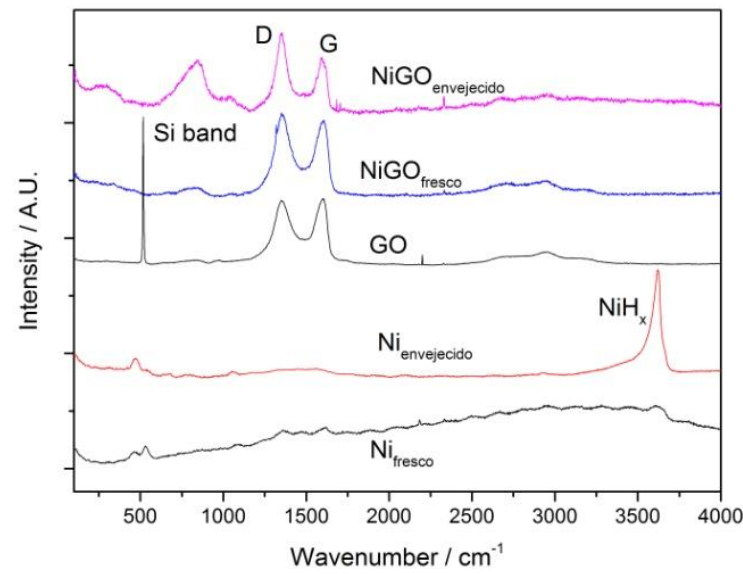

Figura 3: Espectros Raman de los diferentes catalizadores sintetizados antes y después del envejecimiento por cronoamperometría. Se presenta también el espectro Raman de GO comercial.

La espectroscopía Raman es ampliamente utilizada para caracterizar la estructura cristalina, la naturaleza química y defectos en los materiales basados en el grafeno [3]. El proceso de reducción de GO para dar óxido de grafeno reducido (denotado como RGO) puede manifestarse en los espectros de Raman por cambios en la intensidad relativa de dos picos principales, D y G [3]. En la figura 3 se muestran los picos D y G en los espectros Raman de GO comercial y NiGO electrodepositado sobre el electrodo de níquel. El pico D de GO situado en $1349 \mathrm{~cm}^{-1}$ surge del estiramiento de enlace C-C con una intensidad en relación con el tamaño de los dominios $\mathrm{sp}^{2}$ en el plano [12]. El aumento de la intensidad del pico $\mathrm{D}$ es una evidencia de la formación de nuevos dominios $\mathrm{sp}^{2}$. La relación de intensidad relativa de los dos picos $\left(\mathrm{I}_{\mathrm{D}} / \mathrm{I}_{\mathrm{G}}\right)$ es una medida del grado de desorden y es inversamente proporcional al tamaño medio de los grupos $\operatorname{sp}^{2}$ [14]. En literatura se muestra que la intensidad de la relación D/G para RGO es mayor que la de GO porque los dominios formados aumentan el número de clústers $\mathrm{sp}^{2}$ [16]. En nuestro caso, hemos encontrado una $\mathrm{I}_{\mathrm{D}}>\mathrm{I}_{\mathrm{G}}$, teniendo en cuenta los picos Raman integrados, lo que confirma la presencia de RGO en el catalizador NiGO.

Los patrones de difracción de rayos X para Ni-Watts y NiGO muestran claramente las reflexiones típicas esperadas para el níquel con estructura cúbica centrada en las caras (fcc). Ambas muestras no presentan picos correspondientes a impurezas. 
Los valores de $2 \theta$ para los planos $\left(\begin{array}{lll}1 & 1 & 1\end{array}\right)$ y $\left(\begin{array}{lll}2 & 0 & 0\end{array}\right)$ del electrodo de Ni-Watts son $44.5^{\circ}(\mathrm{FWHM}=$ $\left.0.153^{\circ}\right)$ y $51.9^{\circ}\left(\mathrm{FWHM}=0.258^{\circ}\right)$, respectivamente; con una distancia de separación (d) de $2,03 \AA$ para el plano $\left(\begin{array}{lll}1 & 1 & 1\end{array}\right)$ y 1,76 Å para el plano $\left(\begin{array}{lll}2 & 0 & 0\end{array}\right)$. La relación de planos $\left(\begin{array}{lll}1 & 1 & 1\end{array}\right) /\left(\begin{array}{lll}2 & 0 & 0\end{array}\right)$ de Ni-Watts es de 3,2, mientras que en el catalizador de $\mathrm{NiGO}$ es de 5,3, lo que muestra un predominio de planos (1 111 ). La cantidad de planos $\left(\begin{array}{lll}2 & 0 & 0\end{array}\right)$ es menor en el catalizador NiGO que en el Ni-Watts, lo que indica que el catalizador NiGO es más estable [3].

Los electrodos de Ni-Watts y NiGO fueron caracterizados con diferentes técnicas electroquímicas (voltametría de barrido lineal - de sus siglas en inglés LSV- por RDE, voltametría cíclica a diferentes temperaturas, cronoamperometría y espectroscopía de impedancia a diferentes potenciales) en solución $1 \mathrm{M}$ de $\mathrm{KOH}$.

Puede verse que la generación de hidrógeno por cronoamperometría presenta una densidad de corriente más alta en NiGO que en Ni-Watts. La velocidad de pérdida de actividad electroquímica obtenida para el Ni-Watts y NiGO son $4.9 \times 10^{-3} \mathrm{~s}^{-1}$ y $2.2 \times 10^{-3} \mathrm{~s}^{-1}$, respectivamente. Esto implica que el Ni-Watts tiene no sólo una menor actividad electrocatalítica que el NiGO, sino que también se desactiva más rápido debido al proceso de envejecimiento.

En la Figura 3 se muestran los espectros Raman de ta Ni-Watts y NiGO (antes y después de la cronoamperometría). Se puede observar que la muestra NiGO mantiene el espectro Raman después del envejecimiento, mientras que la muestra de Ni-Watts después del envejecimiento muestra la aparición de las bandas correspondientes a los hidruros de níquel, en relación con el proceso de envejecimiento del catalizador y la pérdida de actividad.

La Figura 4 muestra las curvas de LSV para cada catalizador antes y después del envejecimiento a corto plazo medida a $298 \mathrm{~K}$ y $1600 \mathrm{rpm}$. En ambos catalizadores se observa un aumento de la corriente catódica después de envejecimiento, mientras que el potencial de inicio de la reacción de desprendimiento de hidrógeno permanece prácticamente constante. En el caso de catalizador de Ni-Watts envejecido se observa un aumento del $33 \%$ en la densidad de corriente (a -1,5 V vs. SCE). Mientras tanto, el catalizador NiGO envejecido muestra un aumento considerablemente elevado (alrededor del 75\%) en la respuesta electroquímica.

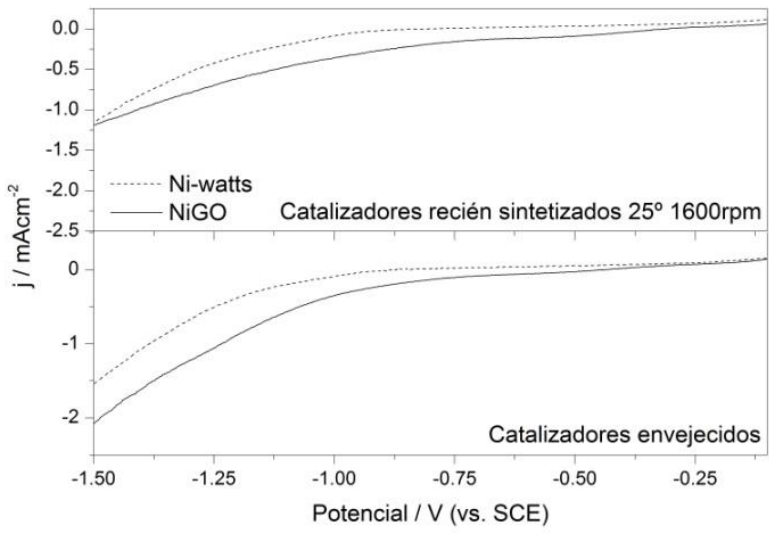

Figura 4: Medidas LSV de catalizadores de niquel Watts y NiGO medidas en catalizadores recién sintetizados y envejecidos.

\section{CONCLUSIONES}

En este trabajo se presenta un método simple y reproducible para la síntesis de catalizadores híbridos de grafeno/níquel y su aplicación a la electrocatálisis de la reacción de desprendimiento de hidrógeno. El método presentado es fácilmente escalable a nivel industrial por lo que puede ser utilizado para mejorar económicamente electrodos de níquel electrodepositados para la generación de hidrógeno.

Se demostró que el GO se reduce durante la síntesis para dar RGO de alta conductividad, disminuyendo de manera significativa la resistencia de transferencia de carga presentada por electrodepósitos de níquel puro.

Se puede ver que la presencia de grafeno en el baño de electrodeposición reduce el número de planos (2 00 ) en la estructura cristalina aumentando la estabilidad, ya que el plano (lllll 11 ) es más estable que el $\left(\begin{array}{ll}2 & 0\end{array}\right.$ 0), lo que podría impedir la formación de hidruros en la superficie de níquel. 
Los estudios Raman sobre catalizadores a los que se los ha envejecido mediante cronoamperometría en $\mathrm{KOH}$ muestra que la superficie del catalizador de NiGO no muestra presencia de hidruros, a diferencia del catalizador de Ni-Watts electrodepositado. Los cambios en el tipo de adsorción de hidrógeno se correlacionan con los resultados obtenidos por espectroscopía Raman que muestran una clara inhibición en la formación de hidruros superficiales debido a la presencia de grafeno en la estructura del catalizador.

\section{AGRADECIMIENTOS}

Los autores agradecen el apoyo financiero de la Agencia Nacional de Promoción Científica y Tecnológica (PICT 1818) y CONICET (PIP 0095). MJG agradece a CONICET por su beca doctoral. EAF, HRC y GIL son investigadores permanentes de CONICET. Los autores agradecen al laboratorio LAMARX por su asistencia en las medidas de SEM/EDS, al laboratorio LANN de INFIQC por las facilidades de microscopía Raman. y al Dr. Raul Carbonio y la Dra. Cecilia Blanco por las medidas de XRD.

\section{BIBLIOGRAFÍA}

[1] BOCKRIS J.O.M, VEZIROGLU T.N. "Estimates of the price of hydrogen as a medium for wind and solar sources” International Journal of Hydrogen Energy, v. 32, pp. 1605-1610, Ago. 2007.

[2] SYMES M.D., CRONIN L. "Decoupling hydrogen and oxygen evolution during electrolytic water splitting using an electron-coupled-proton buffer" Nature Chemistry, v. 5, pp. 403-409, Abr. 2013.

[3] BALL M., WEEDA M. "Hydrogen Use, Safety and the Hydrogen Economy", In: Ball \& Basile \& Veziroglu (eds), The hydrogen economy-Vision or reality?, Compendium of Hydrogen Energy, 1 ed, Volume 4: Woodhead Publishing, 2016.

[4] BAKENNE A., NUTTALL W, KAZANTZIS N. "Sankey-Diagram-based insights into the hydrogen economy of today” International Journal of Hydrogen Energy, v. 41, pp. 7744-7753, May. 2016.

[5] PLETCHER D., XIAOHONG L. "Prospects for alkaline zero gap water electrolysers for hydrogen production”. International Journal of Hydrogen Energy, v. 36, pp. 15089-15104, Nov. 2011.

[6] ZENG K., ZHANG D. "Recent progress in alkaline water electrolysis for hydrogen production and applications." Progress in Energy and Combustion Science, v. 36, pp. 307-326, Ene. 2010.

[7] VIVA F.A., BRUNO M.M., FRANCESCHINI E.A., THOMAS Y.R.J., RAMOS SANCHEZ G., SOLORZA-FERIA O., CORTI H.R. "Mesoporous carbon as Pt support for PEM fuel cell" International Journal of Hydrogen Energy, v. 39, pp. 8821-8826, May. 2014.

[8] HONG Z., LI X., KANG S.Z., QIN L., LI G., MU J. "Modifications of morphology and hydrogen evolution activity for the potassium niobate nanoscrolls by introducing reduced graphene oxide" International Journal of Hydrogen Energy, v. 40, pp. 14297-14304, Nov. 2015.

[9] FRANCESCHINI E.A., LACCONI G.I., CORTI H.R., "Hydrogen evolution kinetics on Ni cathodes modified by spontaneous deposition of Ag or Cu", Journal of Energy Chemistry, Nov. 2016.

[10] FRANCESCHINI E.A., LACCONI G.I., CORTI H.R., "Kinetics of hydrogen evolution reaction on nickel modified by spontaneous Ru deposition: A rotating disk electrode and impedance spectroscopy approach”, International journal of hydrogen energy, V. 41, pp. 3326-3338, Feb. 2016.

[11] MCARTHUR, M.A., JORGE, L., COULOMBE, S., et al., "Synthesis and characterization of 3D Ni nanoparticle/carbon nanotube cathodes for hydrogen evolution in alkaline electrolyte", Journal of Power Sources, v. 266, pp. 365-373, Nov. 2014.

[12] GEIM A.K., NOVOSELOV K.S. "The rise of graphene”, Nature Materials, v. 6, pp. 183-191, Mar. 2007.

[13] RODRÍGUEZ-CARVAJAL J. "Recent advances in magnetic structure determination by neutron powder diffraction", Physica B, v. 195, pp. 55-57, Oct. 1993.

[14] FRANCESCHINI E.A., LACCONI G.I., CORTI H.R., "Kinetics of the hydrogen evolution on nickel in alkaline solution: new insight from rotating disk electrode and impedance spectroscopy analysis", Electrochimica Acta, v. 159, pp. 210-218, Mar. 2015.

[15] SOBON G., SOTOR J., JAGIELLO J., et al., "Graphene Oxide vs. Reduced Graphene Oxide as saturable absorbers for Er-doped passively mode-locked fiber laser" Optics Express, v. 17, pp. 19463-19473, Ago. 2012. 
[16] HALL D.S., BOCK C., MACDOUGALL B.R. "The electrochemistry of metallic nickel: oxides, hydroxides, hydrides and alkaline hydrogen evolution", Journal of Electrochemical Society, v. 160, pp. F235F243, Ene. 2013

[17] GUO Y., SUN X., LIU Y., et al., "One pot preparation of reduced graphene oxide (RGO) or Au (Ag) nanoparticle-RGO hybrids using chitosan as a reducing and stabilizing agent and their use in methanol electrooxidation", Carbon, v. 50, pp. 2513-2523, Jun. 2012.

[18] JORIO A., DRESSELHAUS M., SAITO R., DRESSELHAUS G.F., Raman Spectroscopy in Graphene Related Systems, 1ed, Weinheim, Wiley-VCH, 2011.

[19] QUAINO P., JUAREZ F., SANTOS E., et al., "Volcano plots in hydrogen electrocatalysis - uses and abuses", Beilstein Journal of Nanotechnology, v. 5, pp. 846-854, Jun. 2014. 